\title{
Novel carboxylated PEG-coating on magnetite nanoparticles designed for biomedical applications
}

\author{
Erzsébet Illés ${ }^{1}$, Etelka Tombácz ${ }^{1}$, Márta Szekeres ${ }^{1}$, Ildikó Y. Tóth ${ }^{1}$, Ákos Szabó ${ }^{2}$, Béla Iván ${ }^{2}$ \\ ${ }^{1}$ Department of Physical Chemistry and Materials Science, University of Szeged, Aradi Vt. 1, \\ H-6720 Szeged, Hungary \\ ${ }^{2}$ Department of Polymer Chemistry, Research Centre for Natural Sciences, HAS, P.O. Box \\ 286, H-1519 Budapest, Hungary
}

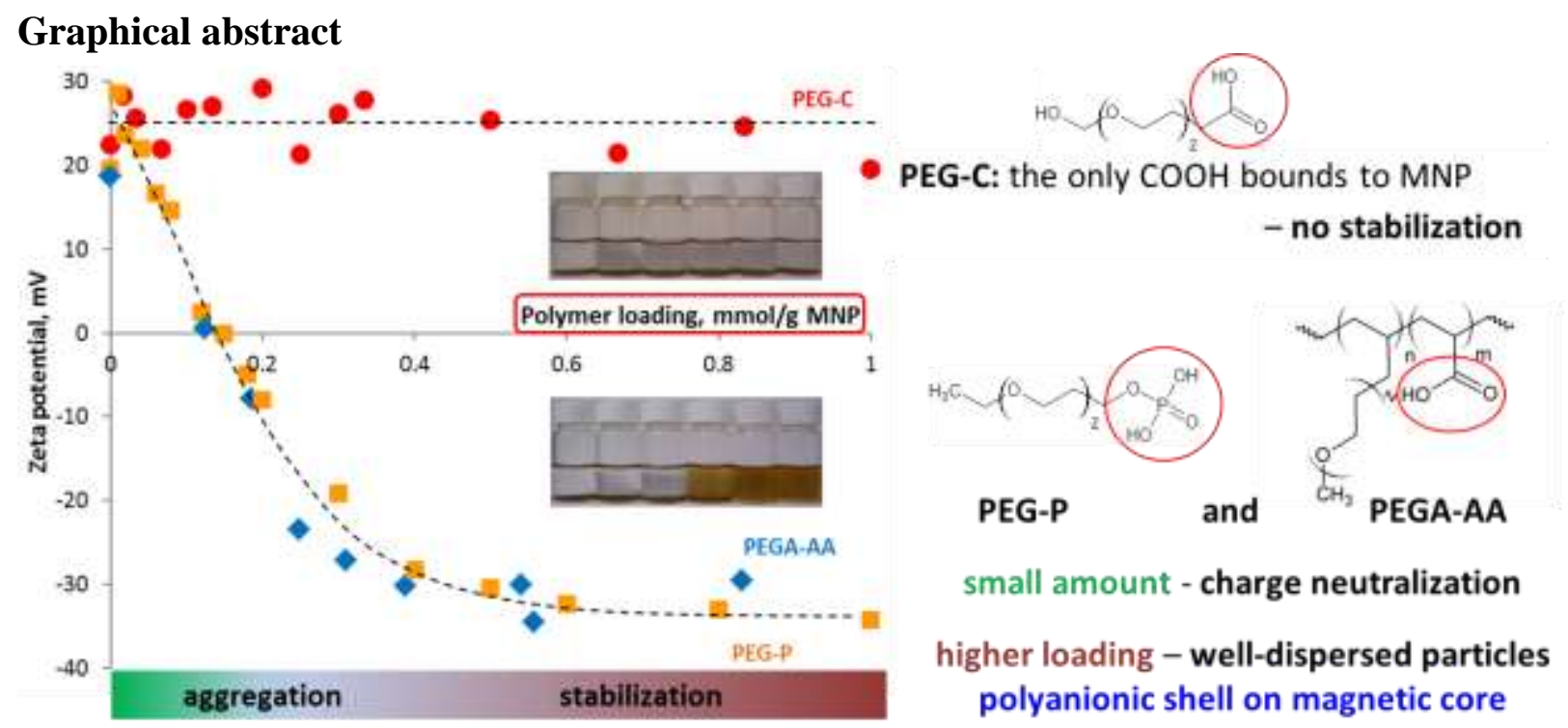

\section{Highlights}

- PEG-polymers were adsorbed through surface complexation on magnetite.

- Increasing load of functionalized PEGs gradually modifies the MNP's surface.

- MNPs coated completely by polyanionic shell can be dispersed in salty media at pH $~ 6.5$.

- Carboxylated comb-like PEG copolymer provides the most efficient biocompatible coating.

\begin{abstract}
Fabrication of PEG coating on magnetite nanoparticles (MNPs) is one of the most favoured ways to ensure biocompatibility. Surface modification of magnetite by an own-prepared comb-like PEG-copolymer (PEGA-AA) was compared with two commercially available ones (carboxy-PEG (PEG-C) and phosphate-PEG (PEG-P)). ATR FTIR data revealed that all polymers form complexes on the surface of MNPs. Electrophoresis and dynamic light scattering (DLS) experiments showed that both the type and quantity of the polymers' anchoring groups influence the aggregation of coated nanomagnets. PEG-C shell does not provide excess negative charges, so magnetite particles became aggregated. However PEG-P and PEGA-AA gradually modify the surface: neutralizing the originally positively charged MNPs below loading $0.5 \mathrm{mmol} / \mathrm{g}$, while above it a polyanionic layer forms on nanomagnets dispersing them in salty media at $\mathrm{pH} \sim 6.5$. The PEGA-AA comb-like copolymer is more efficient for MNPs PEGylation due to the uniform distribution of carboxylates and PEG chains along the carbon skeleton.
\end{abstract}


Keywords: magnetite nanoparticle, PEGylated nanomagnets, superhydrophilic coating, colloid stability, biomedical application

Corresponding authors: E. Illés (+3662544212, illese@ @ chem.hu) and E. Tombácz (+3662544212, tombacz@ chem.u-szeged.hu)

\section{Introduction}

Designing hydrophilic magnetic nanoparticles (MNPs) for diagnostic and/or therapeutic applications, such as MRI contrast enhancement, magnetic hypertermia, drugdelivery, etc. has been in the focus of scientific interest for the last decades [1-7]. Aqueous magnetic fluids (MF) designed for biomedical applications should be non-toxic, biocompatible, chemically stable, and remain uniformly sized even under physiological conditions [8,9]. Bare MNPs (nanomagnets) inherently aggregate in biorelevant media (neutral $\mathrm{pH}$, high salt and protein content) so they cannot be applied in living systems. A protective layer on the particle's surface is necessary to prevent the aggregation, to stabilize the dispersion and to hinder their chemical and biological degradation.

Wide varieties of coating compounds have been published in the literature, among them are frequently used small molecules (e.g. citrate [9-12], surfactants [13-15]) and larger ones (e.g. dextrane [16,17], polyelectrolytes [9,10,18], PEO-PPO-PEO triblock copolymers $[2,19])$ as well. Beside the chemical resistance of the shell, very hydrophilic surfaces are required to avoid the non-specific protein adsorption (opsonisation) in the blood. Recently the PEGylation, i.e. creating protective polyethylene glycol (PEG) (called also as polyethylene oxide (PEO)) layer on MNPs is the most widely applied method to ensure the biocompatibility of nanomagnets [2,20]. The superhydrophilic PEG coating can prevent opsonisation in blood [2,19] and hence it presumably increases the life-time of the PEGylated MNPs in the circulation system. The prolonged circulation of PEG coated MNPs in living systems raises their theranostic potential.

Numerous methods related to the binding of PEG to MNP surface have been described elsewhere [1,2,20-27]. Among several approaches including in situ and post-synthesis coatings, one of the popular ways is to prepare PEG coating on surfacted (mainly oleate double layer covered) nanoparticles [28-31]. Although these PEGylated MNPs exhibit remarkable relaxivity $\left(r_{2}\right)$ values promising for MRI contrast enhancement, the colloidal stability is not satisfactory due the weakly bound top shell of the triple layer coated MNPs [32]. Currently published results proved that the protective layer can be fastened properly on the surface of nanomagnets via functional (e.g. carboxyl, phosphate) groups of organic molecules $[9,24,25,27,31,33]$. Therefore polymers harnessing the joint combination of high hydrophilicity of the PEG chains and anchoring sites that form chemical bonds through carboxylic/phosphate groups open opportunities to construct a new generation of biocompatible core-shell MNPs.

The colloid stability is essential for biomedical applications, since the aggregation of MNPs can lead to embolism or thrombosis in the blood vessels. An optimization procedure of carboxylate@MNP core-shell nanoparticle preparation was established in our group [9], which guides us through a sequence of the physico-chemical tests (e.g. adsorption, electrophoresis and dynamic light scattering measurements) to select the potential candidates for biomedical applications. Briefly only the samples stable under the physiological conditions ( $\mathrm{pH} \sim 7,150 \mathrm{mM} \mathrm{NaCl}$ ) can be chosen for in vitro tests. The steps of this optimization procedure can be used as a guideline to compare the efficiency of PEG-polymers in MNP stabilization. 
The main goal of this paper is to prepare water-based, biocompatible magnetic fluids consisting of core-shell PEGylated MNPs stable under physiological conditions. We compared the adsorption and colloidal stabilizing efficiency of three different PEG-polymers with various structures and anchoring (carboxyl and phosphate) groups on magnetite in order to choose the most efficient PEG-coating.

\section{Materials and methods}

\subsection{Materials}

Magnetite nanoparticles (MNPs) were synthesized by co-precipitation method starting from analytical grade $\mathrm{FeCl}_{2}$ and $\mathrm{FeCl}_{3}$ salts (Molar, Hungary) as described in detail in our previous and recent papers $[8,9,18,32]$. The size of naked nanomagnets is $\sim 10 \mathrm{~nm}$ determined by TEM (picture is not shown here) [9], the material is of black colour and exhibits strong magnetism. The crystalline structure was identified by X-Ray diffraction using the six characteristic peaks of magnetite according to the JCPDS database [34].

Two commercially available linear PEG-polymers with different end groups and an own-synthesized one were chosen for coating the MNPs. The $\alpha$-hydroxy- $\omega$-carboxy PEG (Iris Biotech, Germany) is abbreviated as PEG-C, and the $\alpha$-methoxy- $\omega$-phosphate PEG (Chemicell, Germany) is marked as PEG-P. The PEG-acrylate-acrylic acid comb-like copolymer (PEGA-AA) is synthesized by quasiliving atomic transfer radical polymerisation (ATRP) using a complex of $\mathrm{Cu}(\mathrm{I})$-chloride and hexamethyl-triethylene-tetramine as catalyst. PEGA-AA was purified by passing it through a neutral $\mathrm{Al}_{2} \mathrm{O}_{3}$ column; PEG-C and PEG-P were used as received. The chemical structures of the studied polymers are shown in Fig. 1 and their main features are summarized in Table 1.

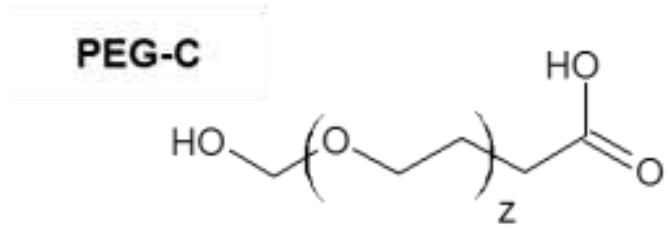

PEG-P<smiles>[Z]C(C)(CCOCC)COP(=O)(O)O</smiles>

PEGA-AA

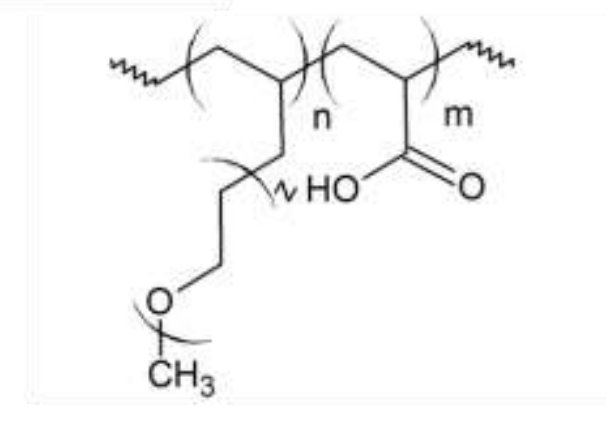

Fig. 1. Chemical structure of the studied PEG-polymers

Table 1. Some characteristics of compounds used for coating MNPs

\begin{tabular}{l|ccc}
\hline $\begin{array}{l}\text { Coating } \\
\text { materials }\end{array}$ & $\begin{array}{c}\mathbf{M}_{\mathbf{n}} \\
(\mathrm{g} / \mathrm{mol})\end{array}$ & $\begin{array}{c}\text { Specific amount of functional } \\
\text { groups* }(\mathrm{mmol} / \mathrm{g})\end{array}$ & Type of compound \\
\hline PEG-C & 3,000 & 0.3 & linear polymer \\
PEG-P & 5,000 & 0.2 & linear polymer \\
PEGA-AA & 4,500 & 5.0 & comb-like polymer \\
\hline
\end{tabular}


*Characteristic functional group is $\mathrm{COOH}$ for PEG-C and PEGA-AA, and phosphate for PEG-P expressed in millimoles per mass of polymer.

All experiments were performed at room temperature $\left(25 \pm 1{ }^{\circ} \mathrm{C}\right)$ and at atmospheric pressure. Considering future biomedical application the coated nanomagnets were studied at near physiological $\mathrm{pH}$. The $\mathrm{pH}$ was adjusted to $6.5 \pm 0.2$ (denoted as $\sim 6.5$ ) to avoid the remarkable carbon dioxide absorption at higher $\mathrm{pHs}$ and the use of various buffers during experiments, which can influence the adsorption equilibrium. This $\mathrm{pH}$ approaches the conditions in cancer cells ( $\mathrm{pH}=6.3-6.8)$ [35].

\subsection{Preparation of PEG-coated core-shell nanomagnets}

The nanomagnets were coated by the adsorption method, equilibrating a given mass of MNP with various amounts $(0-1.5 \mathrm{mmol} / \mathrm{g} \mathrm{MNP})$ of PEG-polymers (PEG-C, PEG-P and PEGA-AA respectively) in the volume of $10 \mathrm{mls}$. The adsorbed amount of PEG polymers is expressed in $\mathrm{mmol} / \mathrm{g}$, where the molar amount of their functional groups is related to the unit mass of magnetite. The $\mathrm{pH}$ was set to $\mathrm{pH} \sim 6.5$ with a $0.1 \mathrm{M} \mathrm{NaOH}$ solution. The samples containing MNPs and polymer were left to stand for an hour (adsorption time). The MFs consisting of core-shell nanoparticles designated as PEG-C@MNP, PEG-P@MNP and PEGA-AA@MNP, were further characterized without any additional purification process and stored in fridge prior to use.

\subsection{Infrared spectroscopy (ATR FT-IR)}

Bio-Rad Digilab Division FTS-65A/896 spectrometer (with DTGS detector) equipped with a Harrick's Meridian Split Pea Diamond ATR accessory was applied to record the ATR FT-IR spectra. The absorbance of the samples was measured in single reflection mode over the 400-4000 $\mathrm{cm}^{-1}$ range (with resolution of $2 \mathrm{~cm}^{-1}$ ), accumulating 256 scans. One drop of the nanomagnets' dispersions (MNP, PEG-C@MNP, PEG-P@MNP and PEGA-AA@MNP) or of the pure polymer solutions (PEG-C, PEG-P and PEGA-AA) was dried onto the surface of the diamond crystal. The polymer loading was $1 \mathrm{mmol} / \mathrm{g} \mathrm{MNP}$, the $\mathrm{pH}$ was set to $\sim 6.5$ and the $\mathrm{NaCl}$ concentration to $10 \mathrm{mM}$. The background spectra were measured on a clean and dry diamond crystal.

\subsection{Electrophoresis measurements}

Zeta potentials of the bare and coated MNPs (MNP, PEG-C@MNP, PEG-P@MNP and PEGA-AA@MNP) were determined using NanoZS (Malvern, UK) apparatus with a 4 $\mathrm{mW}$ He-Ne laser source $(\lambda=633 \mathrm{~nm})$. Electrophoretic mobilities of dilute dispersions $(0.1 \mathrm{~g}$ $\mathrm{MNP} / \mathrm{L}$ ) were measured in DTS 1061 cells at $25 \pm 0.1{ }^{\circ} \mathrm{C}$ and the Smoluchowski equation was used to convert them to zeta potentials. The surface modification effect of the PEGpolymers was studied at various loadings $(0-1.5 \mathrm{mmol} / \mathrm{g} \mathrm{MNP})$ at $\mathrm{pH} \sim 6.5$ and $10 \mathrm{mM} \mathrm{NaCl}$. The $\mathrm{pH}$-dependent surface charging of the nanomagnets was examined between $\mathrm{pH} \sim 3$ and $\sim 10$ set by using $0.1 \mathrm{M} \mathrm{HCl}$ and $\mathrm{NaOH}$ solutions.

\subsection{Particle Size Determination}

The mean diameter of the bare and coated nanomagnets (MNP, PEG-C@ MNP, PEGP@MNP and PEGA-AA@MNP) was measured by NanoZS (Malvern, UK) dynamic light scattering (DLS) instrument operating at $173^{\circ}$ (backscattering mode) at $25 \pm 0.1{ }^{\circ} \mathrm{C}$. Same 
solution conditions were ensured as for electrophoresis experiments $(0.1 \mathrm{~g} \mathrm{MNP} / \mathrm{L}, \mathrm{pH}=3-10$, $10 \mathrm{mM} \mathrm{NaCl}$ ), the amount of the added PEG-polymer was varied between 0 and $1.5 \mathrm{mmol} / \mathrm{g}$ MNP. Aggregation state of the core-shell MNPs was characterized by the change of the average hydrodynamic diameter (Z-Ave). We used the second- or third-order cumulant fit of the autocorrelation functions.

\section{Results and discussion}

\subsection{Adsorption of PEG-polymers on magnetite}

The adsorption of the three different PEG-polymers (PEG-C, PEG-P and PEGA-AA) on magnetite nanoparticles was studied by adding increasing amounts of the compounds. The effect of the increasing PEG-C and PEG-P polymer concentration on particle aggregation can be clearly seen in the following photos (Fig. 2 and 3, respectively). The addition of PEG-C (Fig. 1) did not lead to stable samples even at higher loadings (1.5 mmol/g MNP), the coated MNPs settled from the liquid phase leaving back a clear supernatant. The other two polymers, the PEG-P and the PEGA-AA showed similar behaviour, therefore only the adsorption of phosphate functionalized is presented as an example (Fig. 3). At low polymer concentration ( $<0.5 \mathrm{mmol} / \mathrm{g} \mathrm{MNP}$ ) sedimentation occurred, but both substances were able to stabilize the nanomagnets at larger added amounts, where the proportion of organic compound related to the mass of the solid was high enough ( $>0.5 \mathrm{mmol} / \mathrm{g} \mathrm{MNP})$.

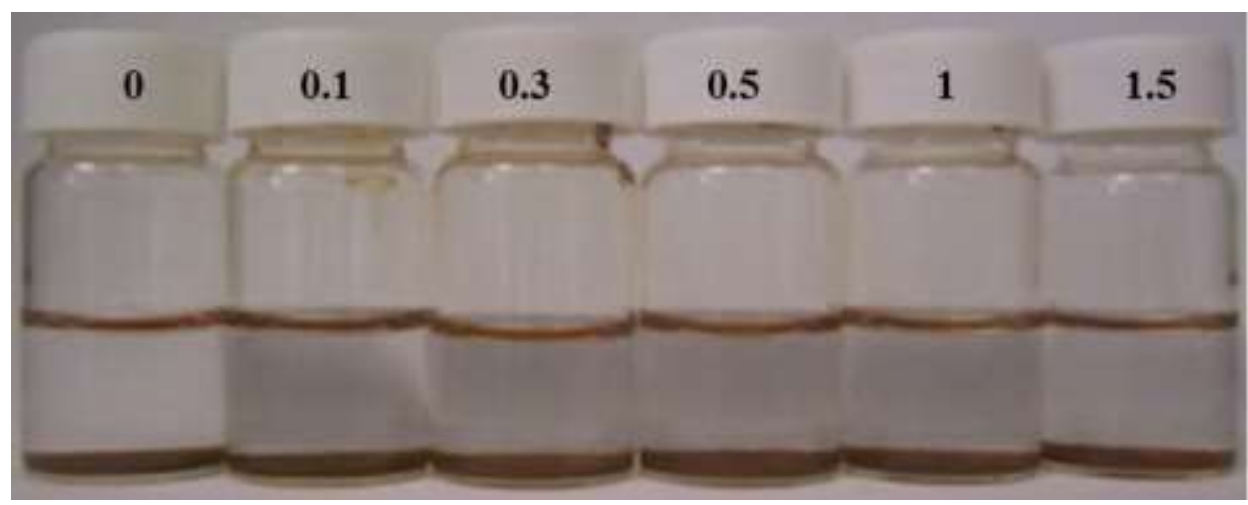

Fig. 2. Adsorption of PEG-C on magnetite at $\mathrm{pH} \sim 6.5$, in the presence of $10 \mathrm{mM} \mathrm{NaCl}$; the amount of polymer increases from 0 up to $1.5 \mathrm{mmol} / \mathrm{g} \mathrm{MNP}$ (from left to right)

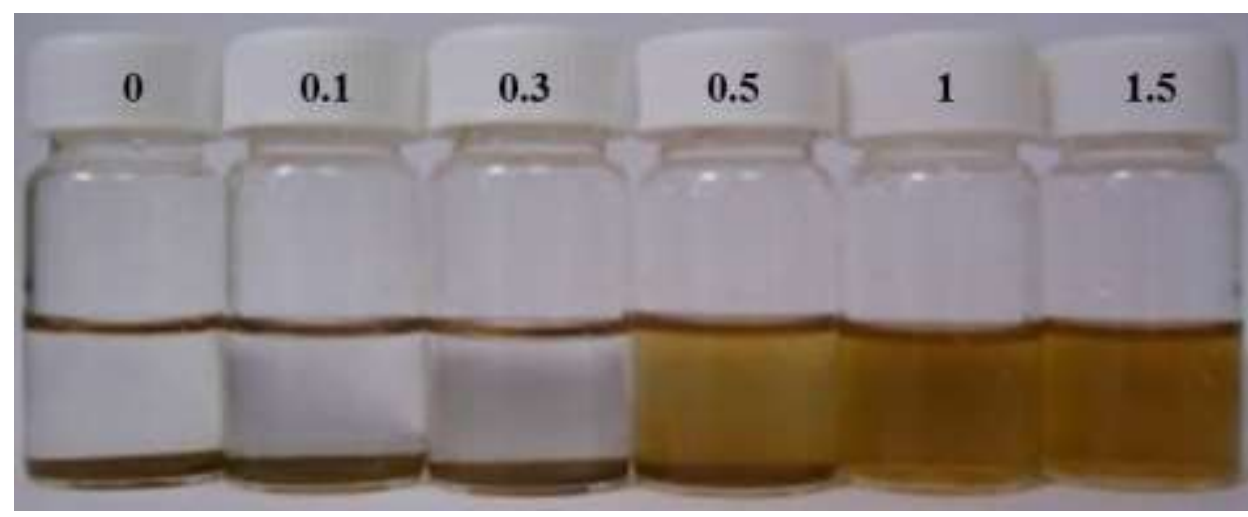

Fig. 3. Adsorption of PEG-P on magnetite at $\mathrm{pH} \sim 6.5$, in the presence of $10 \mathrm{mM} \mathrm{NaCl}$; the amount of polymer increases from 0 up to $1.5 \mathrm{mmol} / \mathrm{g} \mathrm{MNP}$ (from left to right) 
The bonds formed during adsorption of the three PEG-polymers on magnetite surface were studied by ATR FT-IR spectroscopy. In general, the comparison of two characteristic regions (400-800 $\mathrm{cm}^{-1}$ and $900-1900 \mathrm{~cm}^{-1}$ ) of the IR spectra recorded for the coated MNPs and the polymers is used for analysis of binding [9,32]. Since here we focus only on the existence of surface bonds on magnetite's active sites, solely the lower wavenumber regime of the spectra will be discussed. In Fig. 4 , the band at $\sim 555 \mathrm{~cm}^{-1}$ belonging to the $\equiv \mathrm{Fe}-\mathrm{O}$ vibrations $[9,24,25,27,32]$ shifts to higher wavenumbers (from 555 to $558-584 \mathrm{~cm}^{-1}$ ), which indicates that surface complexation occurs, i.e. chemical bond forms between the $\equiv \mathrm{Fe}-\mathrm{OH}$ surface sites and the functional (carboxyl or phosphate) groups of the polymers [24,25,27].

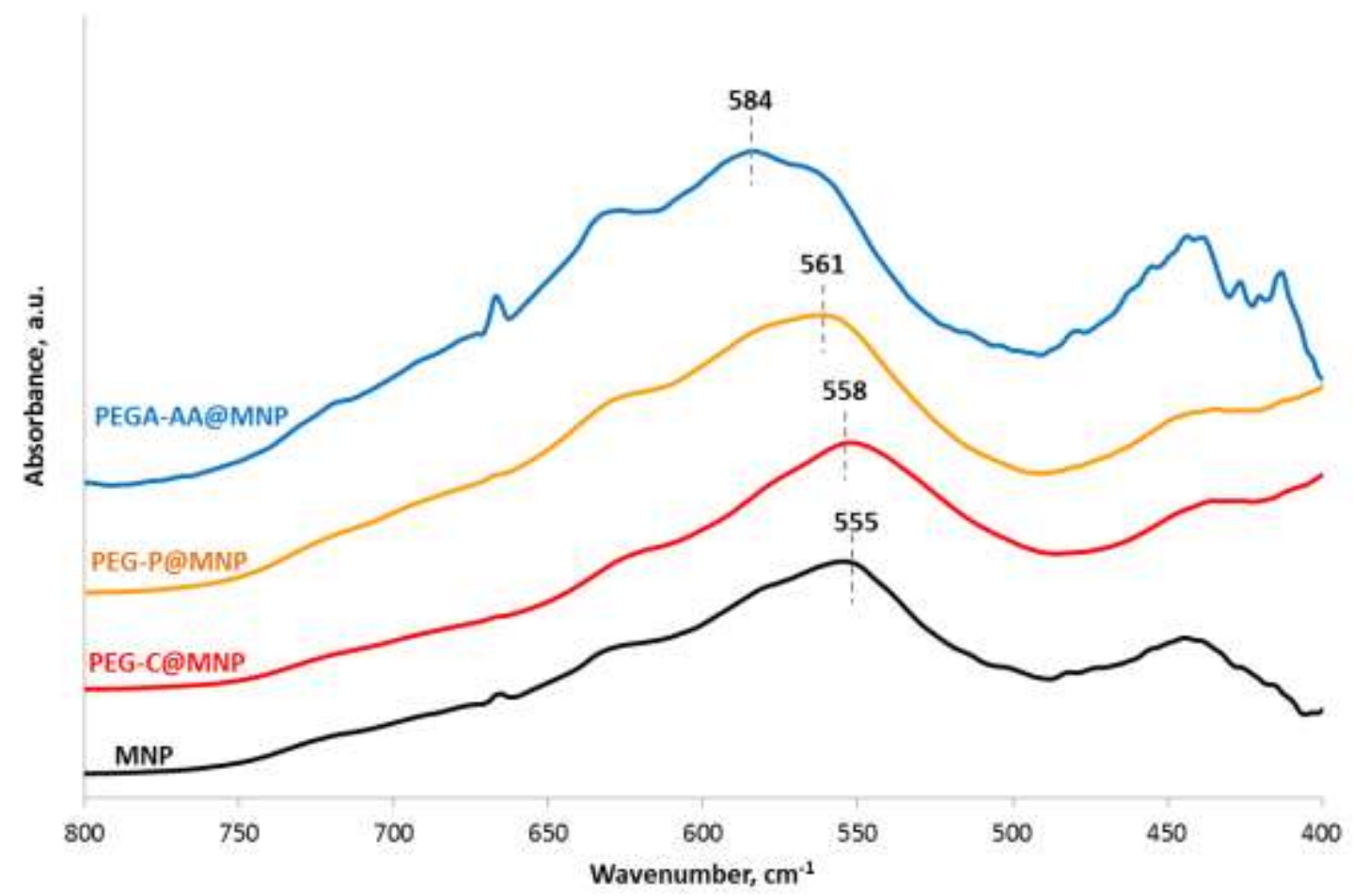

Fig. 4. The characteristic range $\left(400-800 \mathrm{~cm}^{-1}\right)$ of the ATR FT-IR spectra of bare MNP and polymer coated nanomagnets (PEG-C@MNP, PEG-P@MNP, PEGA-AA@MNP). The samples were dried on the ATR crystal from a medium of $\mathrm{pH} \sim 6.5,10 \mathrm{mM} \mathrm{NaCl}$.

Based on our previous results both the protonated and deprotonated forms of the carboxyl moiety can take part in the adsorption leading to H-bond formation, bidentate metal-carboxylate coordination and proton transfer reactions $[8,18]$ on the $\equiv \mathrm{Fe}-\mathrm{O}$ surface sites of magnetite. A small shift of the magnetite-specific Fe-O bond was observed for PEG-C binding, indicating that only few bond forms during adsorption. Since the shift was more convincing for the other two compounds, we can suppose that there are more or stronger bonds between PEG-P or PEGA-AA and the MNP surface sites.

\subsection{Core-shell particle charge}


Electrophoresis experiments showed that the chemically attached PEG-polymers influence the surface charging of nanomagnets depending on the amount of added molecules. As Fig. 5 shows the PEG-C addition did not influence the originally positive zeta potential of the magnetite nanoparticles at $\mathrm{pH} \sim 6.5$, it is $\sim 30 \mathrm{mV}$ even at higher polymer loadings. Since the only carboxyl group of PEG-C is involved in the specific bond formation, there are no more free dissociable groups present providing extra charges for electrostatic stabilization of MNPs. Similar feature was observed for salicylic acid adsorption on alumina [36], where the electrostatic stability also could not be reached as a consequence of the aromatic carboxylic acid baring only one $\mathrm{COOH}$ group.

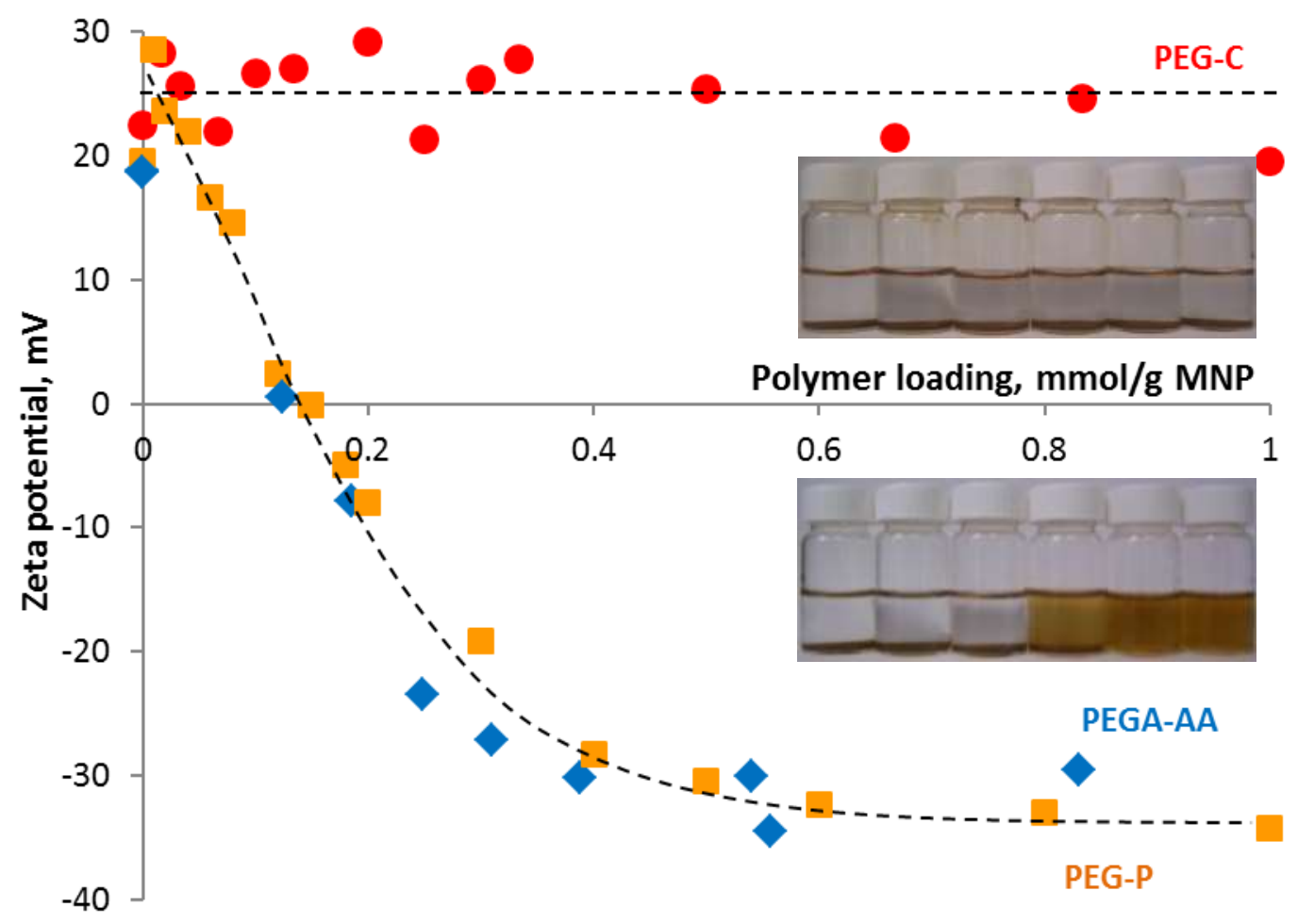

Fig. 5. The zeta potentials of PEG-C@MNPs (red circles), PEG-P@MNPs (orange squares) and PEGA-AA@MNPs (blue diamonds) at various polymer loadings and constant $\mathrm{pH} \sim 6.5$ and 10 mM NaCl. (Inset: upper photo-PEG-C@MNPs and the lower one-PEG-P@MNPs)

On the other hand, an increase in the PEG-P and PEGA-AA adsorption leads to significant lowering of the MNPs' zeta potential (orange squares and blue diamonds in Fig. 5). Increasing the polymer addition, first the charge neutralization of MNP's positive surface charge at $\mathrm{pH} \sim 6.5$ takes place. The isoelectric point (IEP) of the MNPs was reached at $\sim 0.15$ $\mathrm{mmol} / \mathrm{g}$ loading in harmony with our previous experiences $[10,18]$. Complete charge reversal can be achieved (zeta potential became $\sim-35 \mathrm{mV}$ ), if the amount of unbound functional groups is large enough at higher polymer loadings $(>0.5 \mathrm{mmol} / \mathrm{g})$, as it was found by others as well $[26,27]$. Similarly to PEG-C, PEG-P has also only one anchoring group attached at the end of the PEO chain with similar functional group density $(\sim 0.2$ and $\sim 0.3 \mathrm{mmol} / \mathrm{g}$ for PEG-C and PEG-P polymers, respectively, as seen in Table 1), but it is still able to stabilize the MNPs due to the multivalence of the phosphate group. Supposing mono- or bidentate complex formation between $\mathrm{Fe}-\mathrm{O}$ surface sites and phosphate groups take place, free negative charges still remain in the polymer shell providing electrostatic stabilization for nanomagnets. Since 
PEGA-AA is a polycarboxylate decorated with PEO side chains, its polyanionic character at $\mathrm{pH} 6.5$ provides an electrostatic repulsion between the core-shell particles [26,27].

Studying the $\mathrm{pH}$-dependent charge state of the coated MNPs, it can be seen that zeta potential of the PEG-C coated MNPs did not show characteristic difference at various polymer loadings (Fig. 6) compared to the uncoated nanomagnets $\left(\mathrm{pH}_{\mathrm{IEP}} \sim 8\right)$. The apparent increase in zeta potential values may be attributed to a shift of the slipping plane apart from the particle surface due to the presence of an adsorption layer; however, the accuracy of zeta potential measurement $( \pm 5 \mathrm{mV})$ also has to be kept in mind.

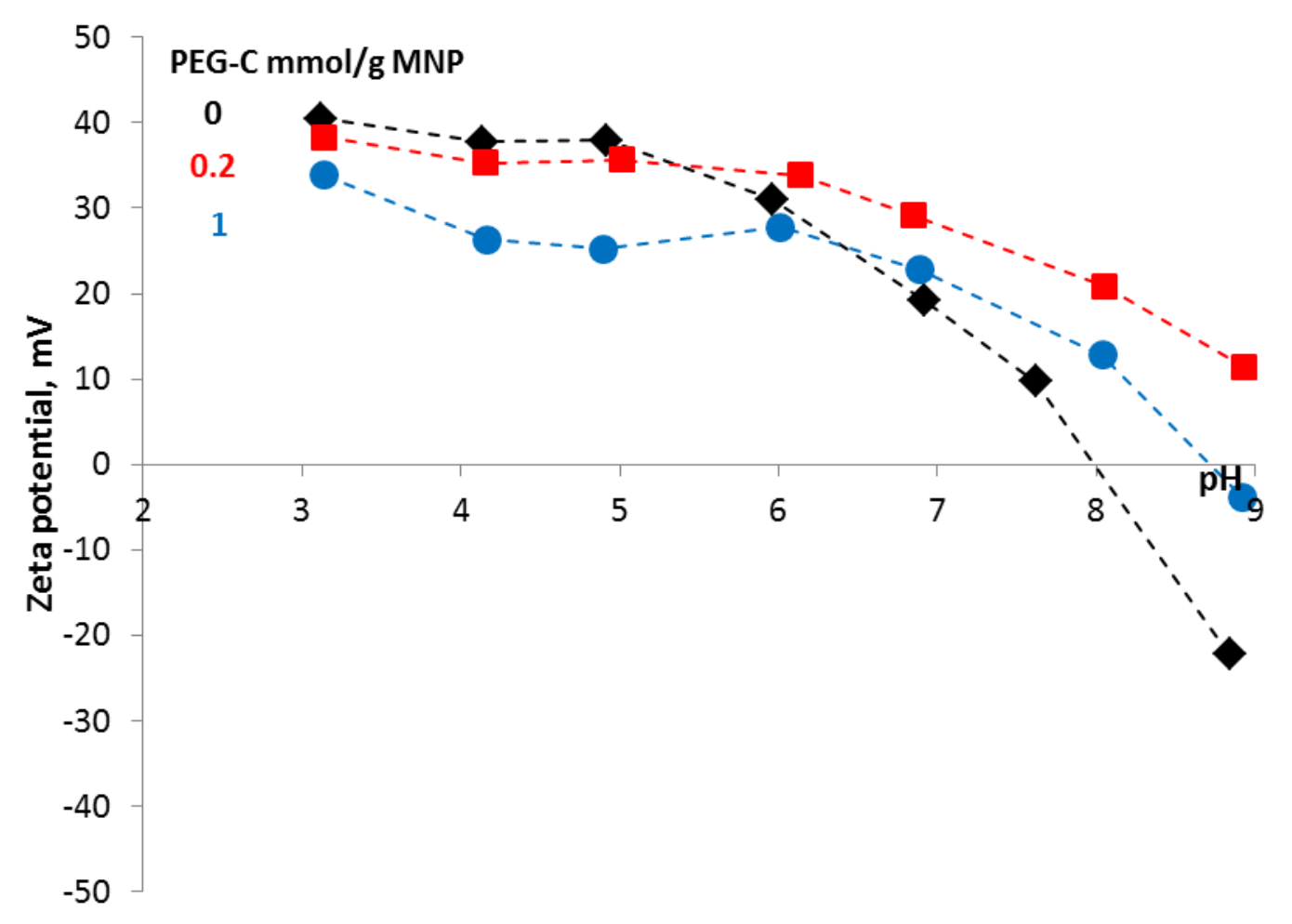

Fig. 6. The pH-dependent zeta potential of naked (black diamonds) and PEG-C coated MNPs at 0.2 (red squares) and 1 (blue circles) $\mathrm{mmol} / \mathrm{g}$ polymer loadings in the presence of $10 \mathrm{mM}$ $\mathrm{NaCl}$ 


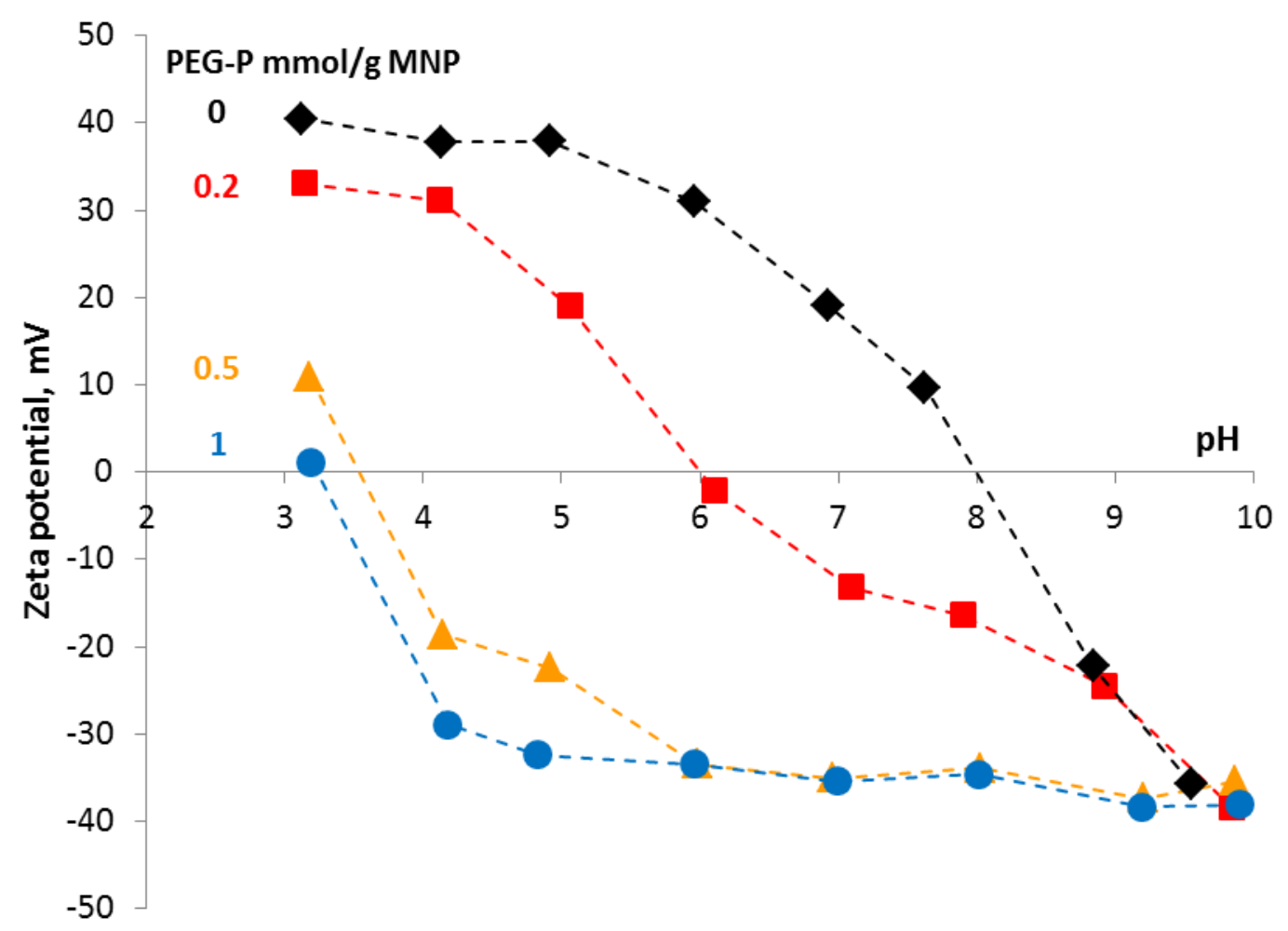

Fig. 7. The $\mathrm{pH}$-dependent zeta potential of naked (black diamonds) and PEG-P coated MNPs at 0.2 (red squares), 0.5 (orange triangles) and 1 (blue circles) $\mathrm{mmol} / \mathrm{g}$ polymer loadings in the presence of $10 \mathrm{mM} \mathrm{NaCl}$

PEG-P and PEGA-AA coated MNPs have similar pH-dependent surface charging behaviour, so only the data set of PEG-P@MNPs are presented in Fig. 7. At small added amount of PEG-P $(0.2 \mathrm{mmol} / \mathrm{g} \mathrm{MNP}$, red squares $)$ zeta potential slightly decreased and the $\mathrm{pH}_{\text {IEP }}$ shifted to a lower $\mathrm{pH}$ (from $\sim 8$ to $\sim 6$ ). An increase in the added amount $(0.5 \mathrm{mmol} / \mathrm{g}$ MNP, orange squares in Fig. 7) of both polymers led to gradual shifting of the $\mathrm{pH}_{\mathrm{IEP}}$ to lower $\mathrm{pH}$ values (from $\sim 8$ to $\sim 4$ ). The further addition of polymer $(1 \mathrm{mmol} / \mathrm{g} \mathrm{MNP}$, blue circles) leads to more negative zeta potentials due to the excess of negative charges originating from the non-bonded acidic groups of the polymers, the minimum value is $\sim-40 \mathrm{mV}$. In addition, the IEP almost disappeared in the studied $\mathrm{pH}$ range $(\mathrm{pH}=3-10)$ as experienced for other polyelectrolyte coatings too $[9,10,26,27]$.

\subsection{Colloid stability}

In biological applications such as intravenous administration, the aggregation of MNPs must be prevented. Testing the colloidal stability under biorelevant conditions is perhaps the most important task. Hydrodynamic diameters of bare (MNP) and coated nanoparticles (PEG-C@MNP, PEG-P@MNP and PEGA-AA@MNP) at various polymer loadings were measured by DLS (Fig. 8). 


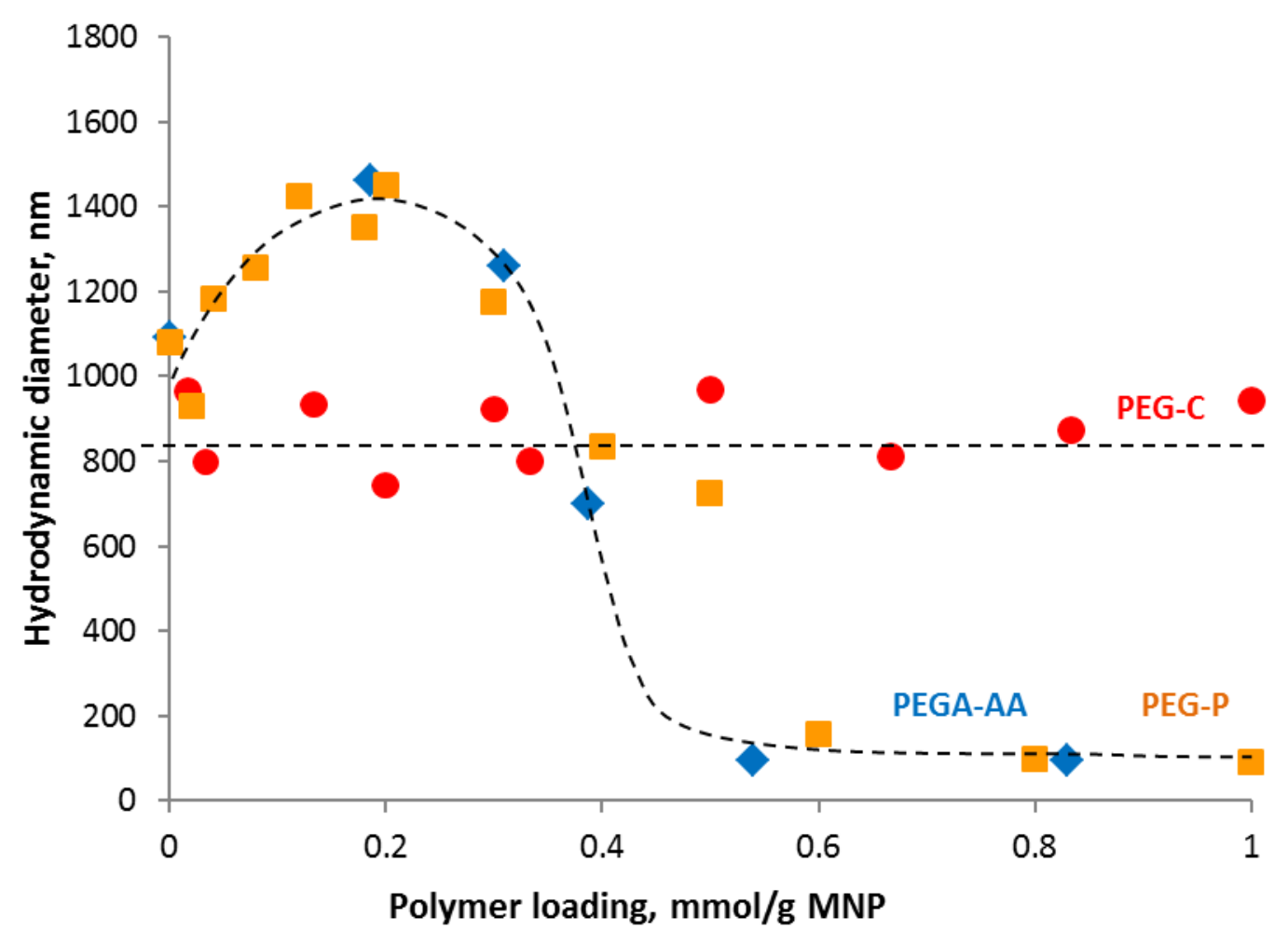

Fig. 8. Hydrodynamic diameter of PEG-C@MNPs (red circles), PEG-P@ MNPs (orange squares) and PEGA-AA@ MNPs (blue diamonds) at various loadings, measured at $\mathrm{pH} \sim 6.5$ and $10 \mathrm{mM} \mathrm{NaCl}$.

The recorded size values characterize the aggregation state of the nanomagnets in harmony with the measured zeta potentials (Fig. 5). PEG-C coated nanoparticles exhibit large mean values $(>800 \mathrm{~nm})$ independently of the added polymer amounts, indicating that aggregation takes place between the non-stabilized MNPs. The samples containing PEG-P (orange squares) and PEGA-AA (blue diamonds in Fig. 8) show different colloidal stability states depending on the polymer loading, since large sizes were recorded at low polymer additions, while small particles were detected at higher degree of coating ( $>0.5 \mathrm{mmol} / \mathrm{g} \mathrm{MNP})$.

In Fig. 9 and 10, the hydrodynamic sizes measured between $\mathrm{pH} \sim 3$ and $\sim 10$ for PEGC@MNP and PEG-P@MNP, respectively, are presented. The naked MNPs show pHdependent aggregation state in harmony with the change of the zeta potential values (see black diamonds in Figures 6 and 7). In the absence of electrostatic stabilization aggregation takes place around the $\mathrm{pH}$ of the IEP $(\sim 8)$ exhibiting a maximum in the measured size values ( $>1000 \mathrm{~nm}$ in Fig. 9). Further from the $\mathrm{pH}_{\mathrm{IEP}}$, however, the magnetite sols are stable, since the particles become positively $(\mathrm{pH}<8)$ or negatively $(\mathrm{pH}>8)$ charged. With increasing amount of PEG-C added to the nanoparticles, no change in $\mathrm{pH}$-dependent aggregation behaviour could be detected even at higher loadings compared to the size values of bare MNPs (Fig. 9). This finding, together with the electrophoresis measurements, supports our idea, that PEG-C is not appropriate for stabilization of nanoparticles at $\mathrm{pHs}$ close to the physiological condition ( 7). 


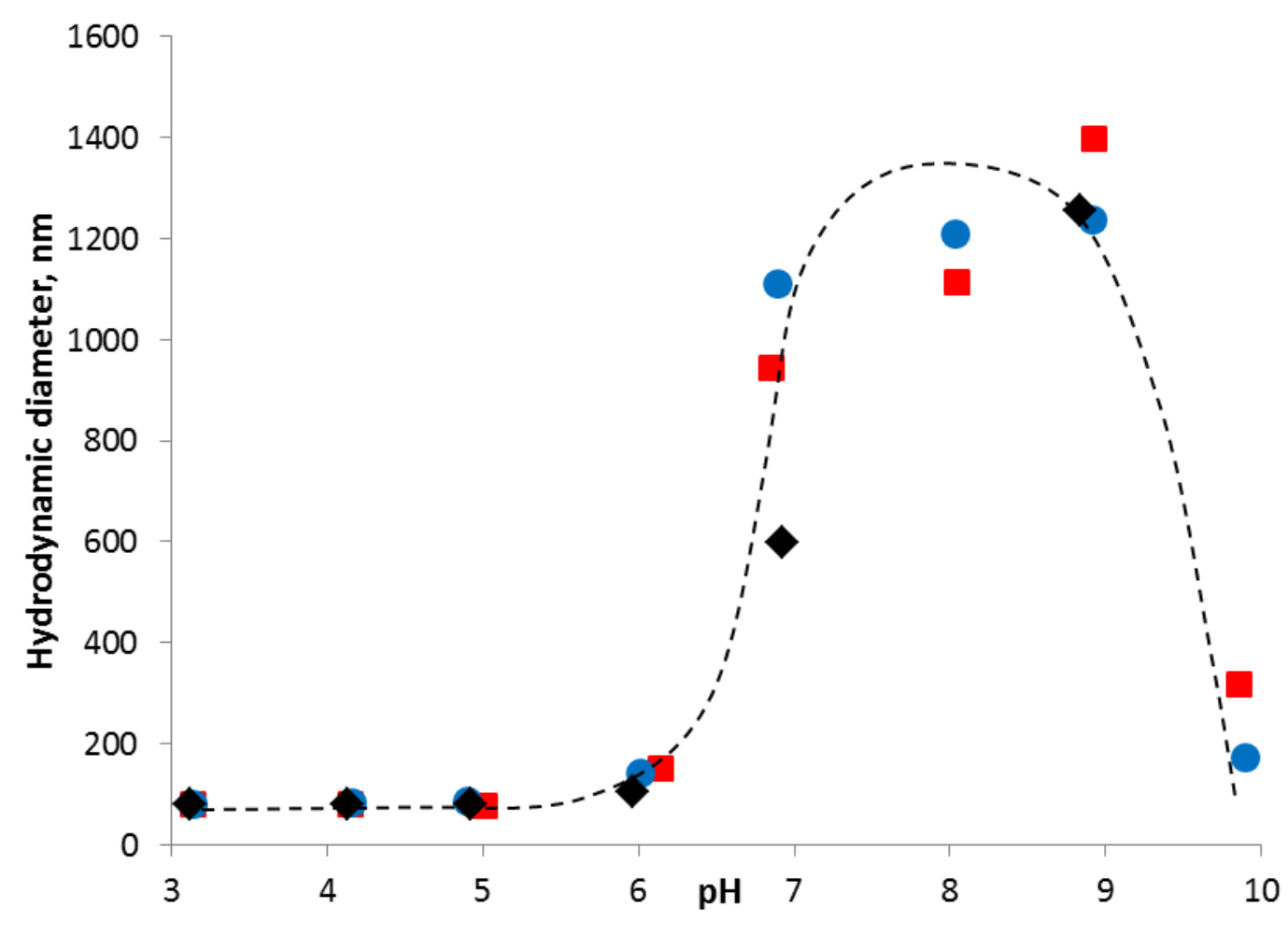

Fig. 9. The pH-dependent hydrodynamic diameter (Z-Ave values) of naked (black diamonds) and PEG-C coated MNPs at 0.2 (red squares) and 1 (blue circles) $\mathrm{mmol} / \mathrm{g}$ MNP loadings in the presence of $10 \mathrm{mM} \mathrm{NaCl}$

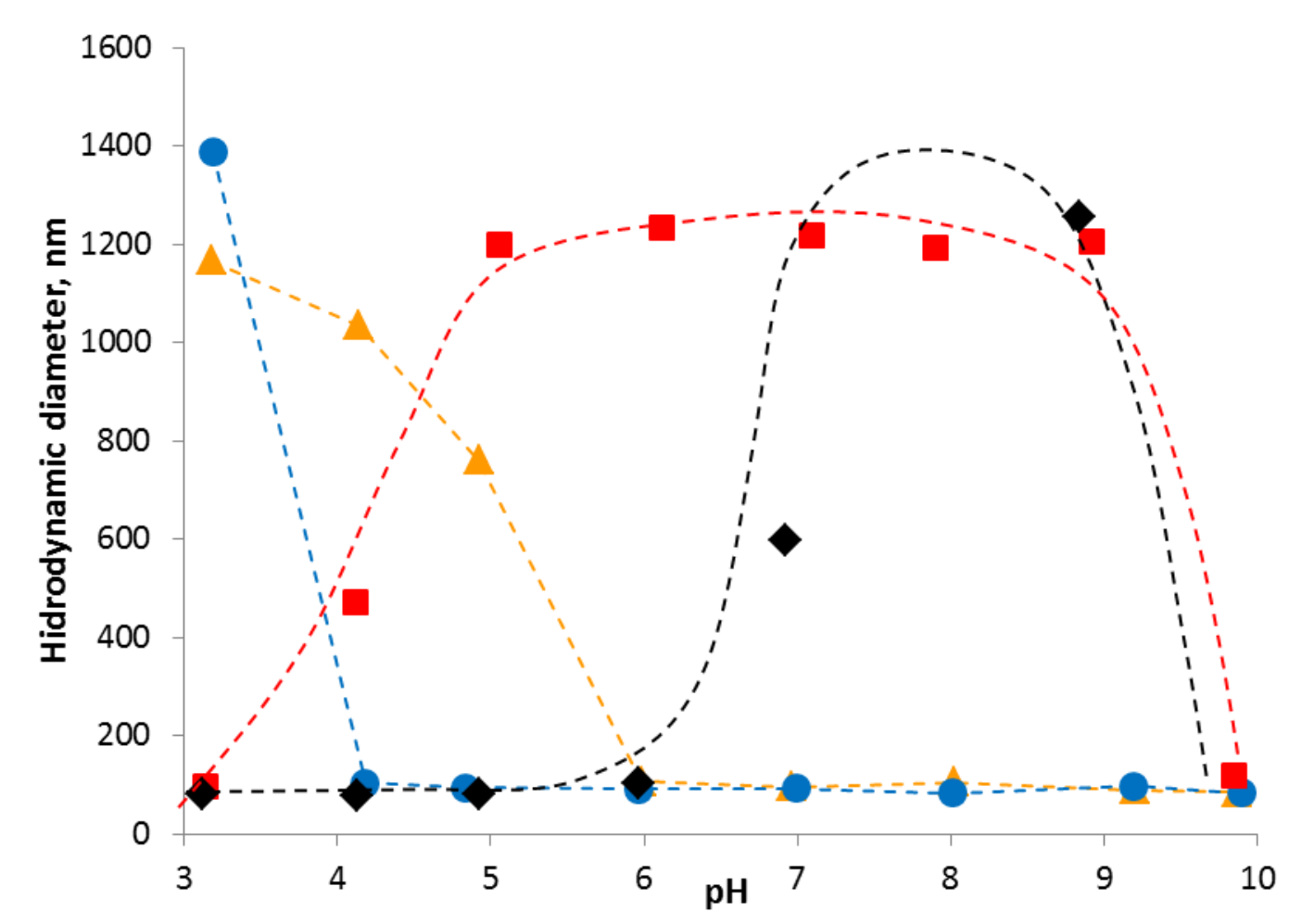


Fig. 10. The $\mathrm{pH}$-dependent hydrodynamic diameter (Z-Ave values) of the naked (black diamonds) and the PEG-P@MNPs at 0.2 (red squares), 0.5 (orange triangles) and 1 (blue circles) $\mathrm{mmol} / \mathrm{g} \mathrm{MNP}$ loadings in the presence of $10 \mathrm{mM} \mathrm{NaCl}$

In contrary, PEG-P and PEGA-AA adsorption significantly influences the $\mathrm{pH}$ dependent aggregation state of MNPs. Since these compounds showed similar behaviour regarding the colloid stability, only the data measured for PEG-P are presented in Fig. 10. Addition of a trace amount of PEG-P resulted in the widening of the aggregation zone with particle sizes larger than $1000 \mathrm{~nm}$ (red squares) due to the partial charge neutralization. An increase in the added amount of both polymers (orange triangles) led to the shifting of the maxima of the aggregation zone to lower $\mathrm{pH}$ values (from $\sim 8$ to $\sim 4$ ) in harmony with the change of $\mathrm{pH}_{\mathrm{IEP}}$ (Fig. 6 and 7). If the polymers were added at a larger amount $(1 \mathrm{mmol} / \mathrm{g} \mathrm{MNP}$, blue circles) stable samples could be obtained, containing particles with an average diameter of $\sim 95 \mathrm{~nm}$. Since significant change (from $80 \mathrm{~nm}$ to $\sim 95 \mathrm{~nm}$ ) could be measured for coated (PEG-P@MNP and PEGA-AA@MNP) particles' size compared to that of the bare nanomagnets, it means, that enough thick layer of polymers formed, and so the steric contribution to the stabilization of MNPs can be assumed. This combined effect, the so-called electro-steric stabilization of nanoparticles was also experienced for other polyelectrolyte coatings $[9,10,26,27]$.

As it was mentioned before, the colloidal stability is essential for MNPs designed for biomedical application. The preliminary results of coagulation kinetic experiments performed by a method published before $[9,32]$ showed that the salt tolerance of the coated MNPs stable at $\mathrm{pH} \sim 6.5$ exceed the physiological salt content $(\sim 150 \mathrm{mM})$. As outlined above both the type and the number of PEG-polymers' anchoring groups determine the aggregation of covered MNPs. It was revealed that multiple charges in the protecting layer can provide appropriate stabilization of nanomagnets. The uniform distribution of carboxylates and PEG chains along the carbon skeleton makes the PEGA-AA comb-like copolymer to be a more sophisticated variant for the PEGylation of MNPs.

The promising physico-chemical results open a new perspective for the biomedical application of these functionalized PEG coated nanomagnets, e.g., MRI contrast enhancement, magnetic hyperthermia and perhaps targeted drug delivery, but to test their real theranostic potential further experiments (e.g. hemocompatibility assay, cytotoxicity tests, MRI contrast enhancement and magnetic hyperthermia measurements) $[9,32]$ are necessary.

\section{Conclusions}

It is probably known, bare magnetite nanoparticles are not suitable for biomedical applications due to the aggregation near the physiological $\mathrm{pH}(\sim 7)$ in the absence of (electrostatic or steric) stabilization. Huge efforts are made to find a coating for MNPs, which fulfills the serious criteria of biocompatibility, and the chemical and the colloidal stability as well. The latter remain untouched; researchers do not pay enough attention to them. Although numerous papers are published in literature related to the MNP coatings they are based on empirical considerations in general. Recently, PEGylation is one of the most widely applied methods to ensure the biocompatibility of nanomagnets. In this paper, our purpose was to provide a qualitative comparison of the colloidal stability of core-shell MNPs bearing various functionalized PEG-polymer shells.

Three functionalized PEG-polymers (PEG-C, PEG-P and PEGA-AA) were used to coat MNPs. Surface modification efficiency of commercially available linear PEGs with one 
functional (carboxyl or phosphate) end-group and an own-synthesized comb-like carboxylated PEG compound was compared. The influence of chemical difference and the number of functional groups present in the PEG-polymer on the colloid stability of MNPs was the fundamental question of this work.

ATR FT-IR measurements proved that these PEG-polymers adsorb by forming chemical bonds on magnetite. It is worthy of attention, that PEG-C, the linear monofunctionalized (carboxylated) PEO polymer was not able to stabilize the magnetic nanoparticles. The only COOH of PEG-C binds to the surface of MNP, so no excess charges are present in the polymer shell providing electrostatic stabilization for nanoparticles.

Other important outcome of the experiments, if these polymers coat the MNPs completely, is that both the addition of PEG-P and PEGA-AA led to colloidally stable samples under near-biorelevant conditions due to the multiple charges linked to each polymer chain. As a conclusion we can state, that beside appropriate loading of polymers, multiple charges are necessary for electrostatic stabilization, originating from either one (phosphate) or more functional groups distributed along the polymer chain (polycarboxylate type structure). Based on these and previous experiences, the continuous distribution of charges in the ownprepared carboxylated comb-like polymer (PEGA-AA) makes it more suitable for stabilization of MNPs. In addition, it opens the possibility for fine tuning of the PEG-polymer shell structure by changing the number and type of functional groups.

\section{Acknowledgement}

This research was supported by the OTKA (NK 84014) grant as well as by European Union and the State of Hungary, co-financed in the framework of TÁMOP-4.2.4.A/ 2-11/1-20120001 'National Excellence Program'.

\section{References}

[1] Q.A. Pankhurst, N.K.T. Than, S.K. Jones, J. Dobson, Progress in applications of magnetic nanoparticles in biomedicine, J. Phys. D: Appl. Phys. 42 (2009) 224001 (15pp).

[2] J.K. Oh, J.M. Park, Iron oxide-based superparamagnetic polymeric nanomaterials: Design, preparation, and biomedical application, Progr. Polym. Sci. 36 (2011) 168-189.

[3] S. Mura, P. Couvreur, Nanotheranostics for personalized medicine, Adv. Drug Delivery Rev. 64 (2012) 1394-1416.

[4] C. Corot, D. Warlin, Superparamagnetic iron oxide nanoparticles for MRI: contrast media pharmaceutical company R\&D perspective. Wiley Interdiscip Rev Nanomed Nanobiotechnology, 2013.

[5] A.S. Wadajkar, J.U. Menon, T. Kadapure, R.T. Tran, J.Yang, K.T. Nguyen, Design and Application of Magnetic-Based Theranostic Nanoparticle Systems, Recent Patens on Biomedical Engineering 6 (2013) 47-57.

[6] S. Dürr, C. Janko, S. Lyer, P. Tripal, M. Schwarz, J. Zaloga, R. Tietze, C. Alexiou, Magnetic nanoparticles for cancer therapy, Nanotechnol Rev 2(4) (2013) 395-409.

[7] M.L. Etheridge, S.A. Campbell, A.G. Erdman, C.L. Haynes, S.M. Wolf, J. McCullough, Journal of Nanomedicine: Nanotechnology, Biology and Medicine 9 (2013) 1-14. 
[8] I.Y. Tóth, E. Illés, R.A. Bauer, D. Nesztor, M. Szekeres, I. Zupkó, E. Tombácz, Designed polyelectrolyte shell on magnetite nanocore for dilution-resistant biocompatible magnetic fluids, Langmuir 28 (2012)16638-16646.

[9] M. Szekeres, I.Y. Tóth, E. Illés, A. Hajdú, I. Zupkó, K. Farkas, G. Oszlánczi, L. Tiszlavicz, E. Tombácz, Chemical and Colloidal Stability of Carboxylated Core-Shell Magnetite Nanoparticles Designed for Biomedical Applications, Int. J. Mol. Sci. 14 (2013) 14550-14574.

[10]E. Tombácz, I.Y. Tóth, D. Nesztor, E. Illés, A. Hajdú, M. Szekeres, L.Vékás, Adsorption of organic acids on magnetite nanoparticles, $\mathrm{pH}$-dependent colloidal stability and salt tolerance, Colloids and Surfaces A 435 (2013) 91- 96.

[11] J. Liu, Z. Sun, Y. Deng, Y. Zou, C. Li, X. Guo, L. Xiong, Y. Gao, F. Li, D. Zhao, Highly Water-Dispersible Biocompatible Magnetite Particles with Low Cytotoxicity Stabilized by Citrate Groups, Angewandte Chemie Int. Ed., 48 (2009) 5875-5879.

[12] R.G. RuizMoreno, A.I. Martinez, R. Castro-Rodriguez, P. Bartolo, Synthesis and Characterization of Citrate Coated Magnetite Nanoparticles, J Supercond Nov Magn (2013) 26:709-712

[13]D. Bica, L. Vékás, M.V. Avdeev, O. Marinica, V. Socoliuc, M. Balasoiu, V.M. Garamus, Sterically stabilized water based magnetic fluids: Synthesis, structure and properties. J. Magn. Magn. Mater. 2007, 311, 17-21.

[14]E. Tombácz, D. Bica, A. Hajdú, E. Illés, A. Majzik, L.Vékás, Surfactant double layer stabilized magnetic nanofluids for biomedical application, J. Phys. Cond. Matter 20 (2008) Article ID 20410 (6 pages)

[15] R.P. Araújo-Neto, E.L. Silva-Freitas, J.F. Carvalho, T.R.F. Pontes, K.L. Silva, I.H.M. Damasceno, E.S.T. Egito, A.L. Dantas, M.A. Morales, A.S. Carriço, Monodisperse sodium oleate coated magnetite high susceptibility nanoparticles for hyperthermia applications, J. Magn. Magn. Mater. 364 (2014) 72-79.

[16] S. Dutz, M. Kettering, I. Hilger, R. Müller, M. Zeisberger, Magnetic multicore nanoparticles for hyperthermia-influence of particle immobilization in tumour tissue on magnetic properties. Nanotechnology 22 (2011) Article ID 265102 (7pp).

[17] Y. Wang, H-Z. Jia, K. Han, R-X. Zhuo, X-Z. Zhang, Theranostic magnetic nanoparticles for efficient capture and in situ chemotherapy of circulating tumor cells, J. Mater. Chem. B 1 (2013) 3344-3352.

[18] A. Hajdú, M. Szekeres, I.Y. Tóth, R.A. Bauer, J. Mihály, I. Zupkó, E. Tombácz, Enhanced stability of polyacrylate-coated magnetite nanoparticles in biorelevant media, Colloids and Surfaces B: Biointerfaces 94 (2012) 242- 249.

[19] V.I. Shubayev, T.R. Pisanic, S. Jin, Magnetic nanoparticles for theragnostics, Adv. Drug Delivery Rev. 61 (2009) 467-477.

[20] B. Mishra, B.B. Patel, S. Tiwari, Colloidal nanocarriers: a review on formulation technology, types and applications toward targeted drug delivery, Nanomed-Nanotechnol 6 (2010) 9-24.

[21]B. Thong-On, B. Rutnakornpituk, U. Wichai, M. Rutnakornpituk, Magnetite nanoparticle coated with amphiphilic bilayer surfactant of polysiloxane and poly(poly(ethylene glycol) methacrylate), J Nanopart Res 14 (2012) 953.

[22] X. Cao, B. Zhang, F. Zhao, L. Feng, Synthesis and Properties of MPEG-Coated Superparamagnetic Magnetite Nanoparticles, Journal of Nanomaterials Volume 2012 (2012) Article ID 607296 (6 pages).

[23] O. Veiseh, J.W. Gunn, M. Zhang, Design and fabrication of magnetic nanoparticles for targeted drug delivery and imaging, Adv. Drug. Deliver Rev. 62 (2010) 284-304.

[24]D. Dorniani, A.U. Kura, S.H. Hussein-Al-Ali, M.Z. Bin Hussein, S. Fakurazi, A.H. Shaari, Z. Ahmad, In Vitro Sustained Release Study of Gallic Acid Coated with 
Magnetite-PEG and Magnetite-PVA for Drug Delivery System, The Scientific World Journal, Volume 2014 (2014) Article ID 416354 (11 pages).

[25] S.R. Kumar, L. Marianna, S. Gianni, A.J. Nathanael, S.I. Hong, T.H. Oh, D. Mangalaraj, C. Viswanathan, N. Ponpandian, Hydrophilic polymer coated monodispersed $\mathrm{Fe}_{3} \mathrm{O}_{4}$ nanostructures and their cytotoxicity, Materials Research Express, 1 (2014) 015015.

[26] C. Barrera, A. Herrera, Y. Zayas, C. Rinaldi, Surface modification of magnetite nanoparticles for biomedical applications, J. Magn. Magn. Mater. 321 (2009) 1397-1399.

[27] A. Masoudi, H.R.M. Hosseini, M.A. Shokrgozar, R. Ahmadi, M.A. Oghabian, The effect of poly(ethylene glycol) coating on colloidal stability of superparamagnetic iron oxide nanoparticles as potential MRI contrast agent, Int. J. Pharm. 433 (2012) 129- 141.

[28] A. Jozefczak, A. Skumiel, Ultrasonic investigation of magnetic nanoparticles suspension with PEG biocompatible coating, J. Magn. Magn. Mater. 323 (2011) 1509-1516.

[29] L. Sun, C. Huang, T. Gong, S. Zhou, A biocompatible approach to surface modification: Biodegradable polymer functionalized super-paramagnetic iron oxide nanoparticles, Materials Science and Engineering C 30 (2010) 583-589.

[30] V. Zavisova, M. Koneracka, M. Muckova, J. Lazova, A. Jurıkova, G. Lancz, N. Tomasovicova, M. Timko, J. Kovac, I. Vavra, M. Fabian, A.V. Feoktystov, V.M. Garamus, M.V. Avdeev, P. Kopcansky, Magnetic fluid poly(ethylene glycol) with moderate anticancer activity, J. Magn. Magn. Mater. 323 (2011) 1408-1412.

[31] M. Mahdavi, M.B. Ahmad, M.J. Haron, F. Namvar, B. Nadi, M.Z.A. Rahman, J. Amin, Synthesis, Surface Modification and Characterisation of Biocompatible Magnetic Iron Oxide Nanoparticles for Biomedical Applications, Molecules 18 (2013) 7533-7548.

[32] E. Illés, M. Szekeres, E. Kupcsik, I.Y. Tóth, K. Farkas, A. Jedlovszky-Hajdú, E. Tombácz, PEGylation of surfacted magnetite core-shell nanoparticles for biomedical application, Colloids and Surfaces A 460 (2014) 429-440.

[33] U.I. Tromsdorf, O.T. Bruns, S.C. Salmen, U. Beisiegel, H. Weller, A Highly Effective, Nontoxic $T_{1}$ MR Contrast Agent Based on Ultrasmall PEGylated Iron Oxide Nanoparticles, Nanoletters 9 (2009) 4434-4440.

[34] R.M. Cornell, U. Schwertmann, The Iron Oxides, VCH,Weimheim, 1996.

[35] K. An, P. Zhao, C. Lin, H. Liu, A pH and Redox Dual Responsive 4-Arm Poly(ethylene glycol)-block-poly(disulfide histamine) Copolymer for Non-Viral Gene Transfection in Vitro and in Vivo, Int. J. Mol. Sci. 15 (2014) 9067-9081.

[36] M. Szekeres, E. Tombácz, K. Ferencz, I. Dékány, Adsorption of salicylate on alumina surfaces, Colloids and Surfaces A 141 (1998) 319-325. 